\title{
La notion de découplage revisitée
}

Decoupling: a concept to clarifie

\section{Laurence Morin-Rivet, Daniel-Mercier Gouin et Maurice Doyon}

\section{(2) OpenEdition}

\section{Journals}

Édition électronique

URL : http://journals.openedition.org/economierurale/2609

DOI : $10.4000 /$ economierurale.2609

ISSN : 2105-2581

Éditeur

Société Française d'Économie Rurale (SFER)

Édition imprimée

Date de publication : 5 mai 2010

Pagination : $3-16$

ISSN : 0013-0559

Référence électronique

Laurence Morin-Rivet, Daniel-Mercier Gouin et Maurice Doyon, «La notion de découplage revisitée », Économie rurale [En ligne], 317 | mai-juin 2010, mis en ligne le 05 mai 2012, consulté le 01 mai 2019. URL : http://journals.openedition.org/economierurale/2609; DOI : 10.4000/economierurale.2609 


\section{La notion de découplage revisitée}

Laurence MORIN-RIVET • Ministère de I'Agriculture, des Pêcheries et de l'Alimentation du Québec (MAPAQ), Direction des études et des perspectives économiques (DEPE)

Laurence.Morin-Rivet@mapaq.gouv.qc.ca

Daniel-Mercier GOUIN • Professeur, titulaire de la Chaire d'analyse de la politique agricole, Université Laval, Québec-dmgouin@eac.ulaval.ca

Maurice DOYON - Professeur au Département d'économie agroalimentaire et des sciences de la consommation, Université Laval, Québec - maurice.doyon@eac.ulaval.ca

\section{Introduction}

$\mathbf{L}^{\mathrm{s}}$ es préoccupations actuelles concernant les formes d'implication de l'État en agriculture ne sont pas nouvelles. Le soutien des revenus des agriculteurs existe dans la plupart des pays industrialisés depuis les années trente, sous diverses formules et combinaisons d'instruments de politique agricole, ceux-ci variant selon les pays et les époques. Depuis 1940 on retrouve dans la littérature économique la préoccupation de rendre ces paiements de transfert le plus efficace et le moins distordant possible, c'est-à-dire de délier les choix de production des agriculteurs de l'aide étatique reçue.

Plus récemment, les préoccupations concernant le soutien interne octroyé par la plupart des pays industrialisés ont été traitées dans le cadre des négociations multilatérales sur le commerce. Dans ce contexte, les pays se sont entendus dans l'Accord sur l'agriculture du cycle de l'Uruguay Round (AAUR) sur le classement des programmes de soutien interne en trois boîtes (ambrée, bleue et verte). Ce classement repose sur le niveau de distorsion que chacun des programmes produit théoriquement sur les échanges et les prix mondiaux.

La boîte verte inclut ainsi les politiques de soutien interne que le régulateur public d'un pays peut utiliser librement, parce que leurs effets sur les échanges sont considérés comme nuls ou, au plus, minimes. Les politiques d'aides découplées se retrouvent dans cette boîte.

Au cours des quinze dernières années, les États ont donc tenté de s'inspirer du concept de découplage dans la réforme des modalités de leur soutien respectif au secteur agricole, notamment afin que ces instruments soient éventuellement inclus dans la boîte verte. Dans la plupart des pays industrialisés, on a donc assisté au passage d'un soutien étatique fortement lié à un produit spécifique (dit « couplé » à la production) à un soutien des revenus moins spécifique à un produit mais davantage basé au revenu global de l'entreprise agricole. Dans certains pays, les réformes ont également mené à un calcul du soutien en fonction d'un historique perçu (dit « découplé »). De ce fait, les politiques classées dans cette catégorie par les différents pays industrialisés ont représenté une part croissante et importante de l'ensemble du soutien à l'agriculture. L'émergence et la réforme des politiques de soutien des revenus tels que le Compte de stabilisation du revenu net (CSRN) et le Programme canadien de stabilisation du revenu agricole (PCSRA) au Canada, les paiements directs états-uniens et les paiements uniques en Europe confirment la nette transformation vers davantage de découplage des aides dans les pays occidentaux.

De ce fait, la définition de ce qu'est une politique découplée devient un enjeu des négociations multilatérales. Mais existe-t-il un consensus, autant dans le débat politique qu'économique, sur ce que l'on entend réellement par «découplage des aides au revenu »?

\section{Découplage : un concept économique ou commercial ?}

Les économistes, les décideurs publics et les négociateurs dans les échanges agricoles 
utilisent tous le concept de découplage dans leurs débats. Toutefois, la signification qui lui est accordée semble différer d'une organisation et d'une profession à l'autre. En fait, le concept du découplage s'insère à la fois dans un discours économique de type académique, ainsi que dans un contexte éminemment politique. De ces multiples utilisations découlent des variantes dans la définition du concept (OCDE, 2001 ; Swinbank et Tranter, 2005 ; Andersson, 2004). Il en résulte deux flous : le premier dans la littérature économique elle-même, qu'elle soit de nature académique ou organisationnelle, et le second, dans l'application du concept dans les négociations multilatérales sur le commerce.

Le concept de découplage est très utilisé, mais existe-t-il une définition consensuelle sur ce qu'il implique, en termes économiques? Dans son opérationnalisation, le concept économique a-t-il été traduit différemment de ses intentions initiales ?

La réponse à ces questions sera traitée en deux temps. Premièrement nous présentons les éléments de définition recueillis dans une revue de littérature effectuée sur le découplage et nous décrivons le flou conceptuel qui en découle, autant dans les écrits de nature politique que dans la littérature économique. Cela nous permet ensuite de faire les deux propositions suivantes:

- La première est qu'il existe un écart considérable entre le concept économique et son application dans la réalité concrète des ententes commerciales internationales sur les échanges agricoles.

- La seconde est que, du point de vue de sa définition économique, le découplage fait référence à une politique qui n'aurait pas d'effet sur la production et les échanges.

Nous proposons dans un deuxième temps de valider ces deux affirmations auprès d'experts internationaux à l'aide de la méthode Delphi. La consultation de différents experts reconnus sur ces questions par le biais de questionnaires de rétroac- tions et l'obtention d'éléments consensuels permet en conclusion de valider nos deux hypothèses, et d'apporter des précisions sur ce que signifie en définitive le concept de découplage des aides au revenu.

\section{Le découplage des aides aux revenus Une notion controversée}

Pour mieux saisir l'intérêt porté à la notion de découplage, il importe de mieux comprendre les mécanismes utilisés pour soutenir la production agricole et leurs effets. Si la distinction entre le soutien des prix et le soutien des revenus est relativement claire dans la littérature économique, celle opposant le soutien des revenus lié au soutien des revenus délié semble nettement moins claire. C'est d'ailleurs une des sources qui alimente la confusion entourant la définition du concept de découplage des aides aux revenus. Nous proposons donc ici les définitions suivantes.

\section{Soutien des revenus lié}

Le soutien des revenus lié implique le maintien d'un revenu cible institutionnel, soit un revenu effectif par unité produite, tout en laissant fonctionner librement le marché. On complète ainsi le prix du marché reçu par le producteur par une subvention à la production (appelée aussi paiement compensatoire), proportionnelle aux volumes offerts. La décision du niveau de revenu adéquat est prise par les pouvoirs publics, et le paiement est ex-post à la décision de production.

Le soutien des revenus lié est souvent présenté comme préférable au soutien des prix - ce dernier modifiant le prix obtenu sur le marché - puisqu'il conduirait à un niveau de distorsion moindre. Les avantages soulignés dans ce type d'intervention sont la mobilité des facteurs de production ainsi que la plus grande proximité du producteur avec les variations et les signaux du marché. 
Cela dit, le soutien des revenus lié est tout de même une aide strictement couplée à la production qui génère une distorsion sur celle-ci (Butault et Le Mouël, 2004 ; Gohin et al., 1999 ; Gouin, 2004). Les producteurs nationaux, bien qu'ils perçoivent la variation du prix mondial, n'en subissent pas de conséquences quant au revenu effectif qu'ils retirent de cette production. Ainsi, un soutien des revenus lié disponible sans limite ne règle pas la propension à la surproduction observée dans le cas du soutien des prix.

\section{Soutien des revenus délié}

On considère un soutien comme non lié, délié ou découplé lorsque la compensation se fait sur la base d'une variation globale du revenu au niveau de l'exploitation complète ou sur une base historique fixe, et non pas en fonction de la variation des revenus relatifs à une quantité de produits et au prix pour une denrée en particulier. Le découplage fait donc référence au passage d'un soutien des revenus lié vers un soutien des revenus délié.

Si l'utilisation du terme découplage est relativement récente, la préoccupation à laquelle il renvoie est présente depuis longtemps dans la littérature : comment résoudre les problèmes suscités par le lien étroit entre les programmes de soutien des prix ou des revenus agricoles et les produits visés par ce type d'intervention? Dans son ouvrage sur la politique agricole états-unienne, Hathaway note, dès 1968 :

\footnotetext{
"The serious problem of resource allocation that arises under most price support and production control programs has led many economists to conclude that the problem of maintaining equitable income in agriculture ought to be separated from the problems of resource allocation. They suggest some kind of direct policy to deal with the problem of farmer's income that does not interfere with either the production of or the demand for farm products. The programs that have been proposed include direct government payments to farmers, income insurance, and modification of the tax structure" (Hathaway, 1968).
}

Bien qu'Hathaway énonce ici le principe d'une politique de soutien des prix qui interfère avec les choix de production, et donc qui n'est pas découplée, les suggestions qu'il mentionne pour résoudre le problème ne conduisent pas nécessairement à des programmes déliés. En effet, un programme de paiements directs peut très bien être un programme lié. D'autres ouvrages (Brandow, 1955 cité par Gilson, 1988 ; Little et al., 2004) abordent le concept de découplage. Or, ces ouvrages ne fournissent pas une définition claire du découplage. Les programmes qui y sont discutés relèvent de paiements directs qui correspondent davantage à la définition du soutien des revenus lié, étant donné le lien qu'ils continuent d'avoir avec le nombre d'unités produites ou le type de produits.

Il est également intéressant de noter que la plupart des références faites à des programmes de soutien des revenus unitaires qualifient ces derniers de « découplage partiel ». La liste des auteurs abordant les paiements directs comme étant du découplage pourrait être très longue (Bergman, 1980 ; Koester et Tangermann, 1977). Nous n'utilisons pas dans cet article la classification de découplage partiel puisqu'elle contribue à alimenter la confusion entre le concept de découplage et son application. Dans la section qui suit, nous présentons de quelle façon le découplage des aides aux revenus s'est imposé dans la littérature économique et comment les frontières du concept y sont demeurées relativement floues.

\section{Le concept de découplage dans la littérature économique : une définition à préciser}

Dès 1945, on retrouve des écrits qui amorcent la réflexion sur ce que nous appellerons plus tard le découplage. Cette année-là, l'American Farm Economic Association offre de primer les meilleures études dans le cadre d'un concours sur le thème suivant : «Une politique de prix agricoles compatibles avec le progrès économique et susceptible de 
favoriser des revenus agricoles plus adéquats et plus stables » (Nicholls et Johnson, 1946, cités par Gilson, op. cit.).

Ces études sont unanimes sur un aspect en particulier : la principale fonction des prix agricoles est de refléter les choix des consommateurs quant à la répartition des ressources agricoles entre les divers produits.

Quelques années plus tard, Swerling (1959, cité par Hathaway, op. cit.) propose un programme de soutien des revenus en huit points visant à pallier leur chronique instabilité et qu'il nommera l'assurance-revenu. L'assurance-revenu de Swerling est une forme de paiements directs basés sur un historique de revenus, au lieu d'être basé sur les prix courants d'un produit en particulier. Cette proposition ne recevra pas beaucoup d'attention de la part du monde politique, même si cette dernière gagne le prix de l'American Farm Economic Association. Il s'agit en fait d'une proposition de découplage des aides très similaire aux conditions des programmes de soutien des revenus mis en place au cours des dernières années au Canada.

En Europe, Quaden aborde aussi la question des programmes découplés dès 1973 : «Une idée commence à faire son chemin : substituer le soutien direct des revenus au soutien des prix, le soutien direct des personnes au soutien des produits ». Et dans le paragraphe suivant, Quaden définit ce que pourrait être un tel programme :

"Quelques commentateurs ayant [...] proposé de réduire sérieusement le niveau moyen des prix agricoles, ont suggéré, pour compenser cette baisse, d'accorder simultanément des paiements directs [...]. Ces indemnités compensatoires n'auraient cependant rien à voir avec les deficiency payments jusqu'ici pratiqués en Grande-Bretagne 1 , qui, comme le soutien des prix, présentent le caractère d'une prime à la production. La formule la plus souvent avancée consiste dans l'attribution d'aides viagères calculées d'après les surfaces affectées à l'agriculture dans les années précédant la décision de baisse des prix, aides dont le taux de base irait diminuant au-delà d'une certaine dimension de l'exploitation [...]» (Quaden, 1973).

1. Un programme de soutien des revenus unitaires, donc lié.
Bien qu'il n'utilise pas les mêmes termes, on peut assimiler les indemnités compensatoires de Quaden à ce que l'on entend aujourd'hui par un soutien des revenus délié ${ }^{2}$.

Le contexte de surproduction, l'accumulation de stocks de denrées et l'explosion des dépenses étatiques de soutien à l'agriculture au début des années 80 conduisent à relancer le questionnement des systèmes d'intervention en place et leurs effets de distorsion. Les premières tentatives de définition du terme « découplage » datent donc des années 1987 à 1988 (soit les années précédant la signature de l'AAUR). Certaines de ces définitions présentent des caractéristiques assez précises de ce que devrait être le découplage. Roberts $e t$ al. (1999) décrivent le découplage ainsi " breaking the links between support and key market variables including production, prices and input use ». Le découplage devrait donc certainement comprendre la notion de suppression du lien entre les paiements et la production en tant que tel.

En ce sens, la plupart des auteurs relèvent comme principale caractéristique le fait que ce type de politique n'a pas d'influence sur les décisions de production des producteurs agricoles (Warley, 1988 ; Carr et Rossmiller, 1988 ; Boschwitz, 1987 ; Cahill, 1997 ; OCDE, 2001 ; Ray, 2002 ; Burfisher et Hopkins, 2004). Le découplage brise le lien entre l'éligibilité au paiement et les actions du producteur en termes de choix de production. D'autres soutiennent plus globalement que le découplage ne crée pas de distorsion sur la production, ce qui renvoie sensiblement à la même idée (Moschini et Sckokai, 1994 ; Schmitz et al., 2003).

Un autre point commun à certains auteurs est qu'une politique découplée n'entraîne pas de changement au niveau de la demande des consommateurs (Gilson, 1988 ; Moschini et Sckokai, 1994) ou comme l'écrit Warley

2. Toutefois, plutôt que ce type de programme de soutien des revenus délié, Quaden préférait plutôt un "régime général et permanent de revenu minimum garanti à chaque citoyen ». 
(1988) : « La seule condition essentielle est que les paiements de transfert soient faits d'une façon qui n'influe pas sur les décisions de production des agriculteurs ni sur les achats des consommateurs ».

On peut aussi souligner que le découplage doit permettre une libre détermination des prix par les forces du marché (Cahill, 1997 ; OCDE, 2001). Cette précision est importante car le libre-marché constitue souvent la référence sur laquelle les auteurs se basent pour justifier et définir le découplage. Burfisher et Hopkins (op. cit.) abondent dans le même sens : "Decoupling is sometimes used to refer to the increased market orientation of farm programs ».

Un autre aspect commun à de nombreuses définitions est l'effet d'une politique découplée sur l'allocation des ressources, que ce soit par rapport à la production d'une denrée en particulier ou à des pratiques de production. Selon cette définition, les ressources affectées à la production agricole (et à la consommation) le sont par des forces compétitives de la demande et de l'offre sur le marché. À cet effet, Gilson mentionne :

"Il y a découplage lorsque le paiement d'appoint public, la subvention à la production, l'intervention sur les prix, ou toute forme d'aide ou d'avantages financiers publics [...] dont profite un producteur agricole n'a pas d'incidence ni d'influence directe sur l'affectation des ressources (au niveau de la production ou de la consommation) consacrées à un produit en particulier ou encore à une pratique de production relative à un produit précis» (Gilson, 1988).

Ces différentes précisions amènent, selon les auteurs, à une autre caractéristique du découplage. Warley (op. cit.) et Moschini et Sckokai (op. cit.) notent que le découplage ne crée pas de distorsion globale sur le commerce. L'OCDE conclut dans le même sens : «Théoriquement, une politique totalement découplée ne devrait pas avoir d'effet commercial au-delà des frontières $d u$ pays $\gg($ OCDE, 2001).

Plusieurs autres auteurs font référence au fait qu'une politique découplée n'entraîne pas de variation du niveau des

\section{Figure 1. Recension des écrits sur le concept économique du découplage}

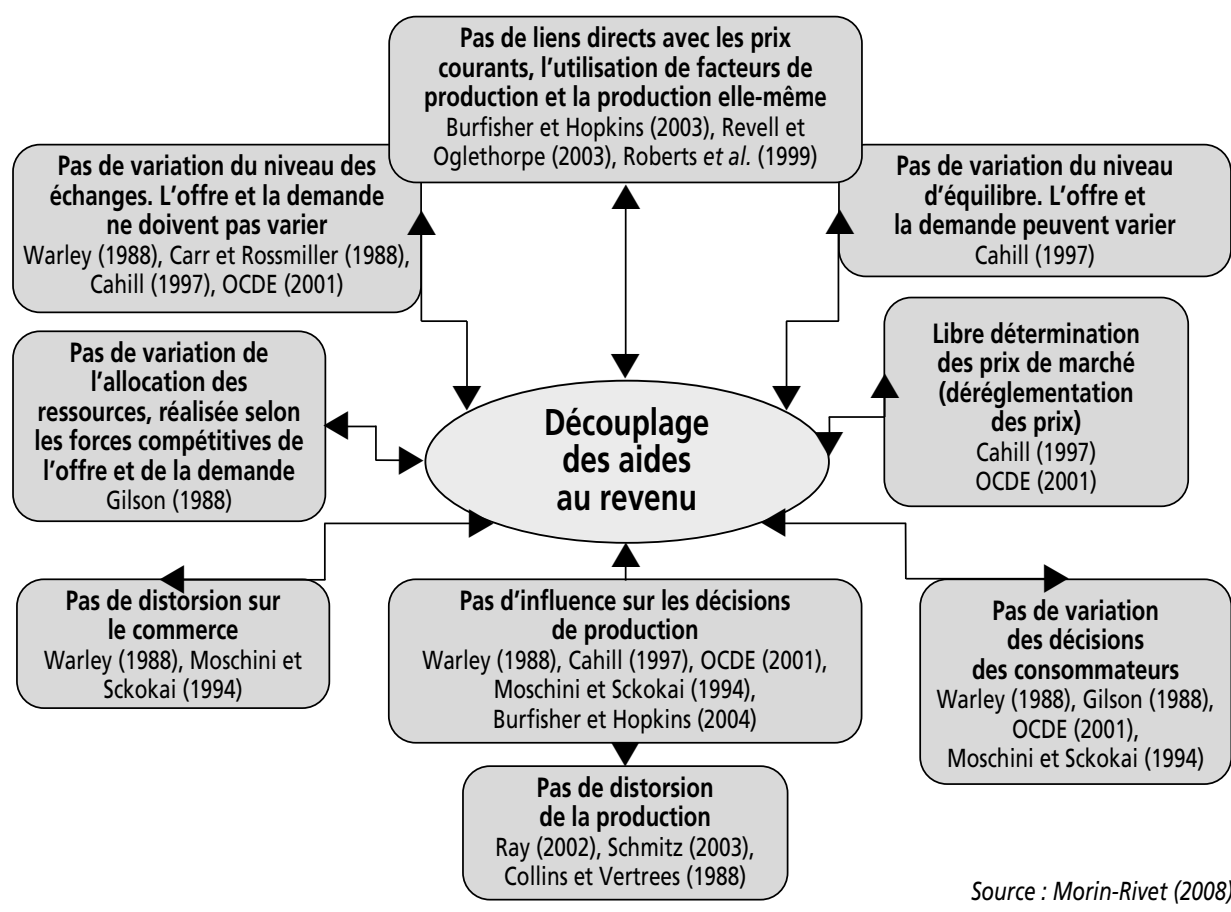


échanges, c'est-à-dire que les courbes d'offre et de demande d'un produit en particulier ne devraient pas varier avec ce type de politique (Warley, 1988 ; Carr et Rossmiller, 1988 ; Cahill, 1997 ; OCDE, 2001).

Une autre approche vise à statuer que le découplage, au contraire de la définition précédente, pourrait faire varier la forme des courbes d'offre et de demande d'un produit (Andersson, 2004). Toutefois, une politique découplée ne ferait pas varier le niveau d'équilibre du marché d'un produit (Cahill, op. cit.). C'est cette approche qui a été reprise depuis par l'OCDE.

La figure 1 résume les principales caractéristiques du terme découplage recensées dans la littérature économique.

\section{Le découplage en pratique}

En pratique, on peut également classer les définitions du découplage selon deux approches (Swinbank et Tranter, 2005 et Andersson, 2004), l'ex-ante et l'ex-post.

- La définition ex-ante du terme découplage est basée sur des critères, sans aucune garantie toutefois que le soutien n'influencera pas la production de façon ex-post. On fait ici référence principalement au «policy design », c'est-à-dire à une approche qui s'attarde davantage à la conception d'une politique qu'à l'application d'un concept théorique pur. C'est dans cette catégorie que l'on retrouve la définition du découplage utilisée à l'OMC et à laquelle un grand nombre d'auteurs (notamment Guyomard et al., 2000 ; Swinbank et Tranter, 2005) se réfèrent directement lorsqu'ils définissent le terme de découplage. La boîte verte de l'AAUR énumère cinq critères que les paiements doivent rencontrer pour être considérés comme découplés : les auteurs qui l'utilisent prennent intégralement les critères énoncés dans l'Accord comme étant LA définition de ce qu'est le découplage. Or, il s'agit d'une application du concept qui relève d'un texte institutionnel issu de négociations entre les acteurs ${ }^{3}$. Selon cette approche, le montant des versements de soutien ne sera fonction ni du type ou du volume de production, ni du prix à la production, ni des facteurs de production employés. Finalement, le soutien ne sera pas conditionnel à une obligation de produire. Cette approche, rend opérationnel le concept économique du découplage. De façon similaire, Burfisher et Hopkins (2003) optent aussi pour une définition ex-ante qui stipule que : «Only subsidies that do not depend on current prices, factor use, or production can be considered fully decoupled from farm production decisions ».

- La seconde approche, celle du ex-post, est basée sur ce qui survient après la mise en place de la politique, sur les conséquences et les résultats qu'elle implique. "The ex-post definition is consequently based on the outcome of the policy» (Andersson, 2004). L'OCDE rejoint cette approche dans sa definition : «A policy is fully decoupled if it does not influence production decisions of farmers receiving payments and if it permits free market determination of prices » (OCDE, 2001). À ce titre il est intéressant de noter que le point 1 de l'Annexe 2 de l'AAUR, qui définit les deux critères de base des soutiens exempts des engagements de réduction, fait davantage référence aux impacts ex-post du soutien interne dans sa mise en contexte ou, autrement dit, aux effets d'une politique sur les échanges.

\section{Point 1}

Les mesures de soutien interne qu'il est demandé d'exempter des engagements de réduction répondront à une prescription fondamentale, à savoir que leurs effets de distorsion sur les échanges ou leurs effets sur la production doivent être nuls ou, au plus, minimes [...] (OMC, Annexe 2 de l'AAUR).

3. On fait référence ici au point 6 (soutien interne) de l'Annexe 2 de l'AAUR qui définit les conditions pour qu'un soutien soit considéré comme découplé. 
La définition de l'OMC comporte donc des éléments des deux approches, la finalité à atteindre étant la minimisation des « effets de distorsion sur les échanges », alors que les moyens pour y parvenir relèvent d'une liste de critères. En revanche, c'est essentiellement de l'approche ex-post que relève la définition de Cahill, que l'OCDE a adoptée pour la poursuite de ses travaux sur le découplage.

Tel qu'Andersson le note, le fait qu'il existe deux approches induit un certain dédoublement de l'utilisation du mot découplage, qui varie selon les acteurs. L'utilisation de l'une ou l'autre approche détermine en partie les résultats obtenus dans les études sur le découplage. "A study that defines a policy as being decoupled based on criteria, and thus independant of its effect on output can, in a an ex-post econometric analysis of this policy reach the conclusion that the decoupled policy have affected the production decision " (Andersson, 2004). Selon cet auteur, une politique peut donc être classée comme découplée selon un des deux types de définitions, et couplée selon l'autre, ce qui démontre l'importance de clarifier la définition choisie lorsqu' on étudie le concept, mais également qui justifie la nécessité d'un certain consensus dans la signification que l'on donne au découplage.

Le bref historique de l'apparition du concept de découplage dans la littérature économique scientifique et appliquée démontre que les recherches d'une reformulation des programmes de soutien à l'agriculture ne datent pas d'hier. Toutefois, malgré toutes ces années de discussions, on remarque un faible consensus existant sur la définition même du concept (Beard et Swinbank, 2001). Des programmes utilisant une base unitaire pour la compensation se réclament souvent du découplage, même si leur application relève pourtant d'une autre catégorie de soutien des revenus, soit le soutien lié. Si plusieurs auteurs admettent la multiplicité des définitions, ils s'enten- dent généralement sur les raisons qui poussent à la reformulation des aides aux revenus, soit principalement la nécessité de réduire la distorsion causée par les mécanismes d'intervention traditionnels. Ils considèrent qu'une grande difficulté persiste toujours dans la recherche d'une définition opérationnelle. Carr et Rossmiller soulignent :

"De façon générale, le terme désigne un transfert de revenu en faveur des agriculteurs, [...] qui n'influencerait pas les choix de production et n'aurait probablement aucun effet sur les échanges commerciaux. Comme on emploie souvent le terme sans le définir avec précision, il règne une certaine confusion au sujet des rapports entre le découplage et les diverses formes de programmes de subventions agricoles appliquées dans différents pays » (Carr et Rossmiller, 1988).

L'OCDE (2001) abonde également dans ce sens : «The word decoupling is widely used, with various interpretations depending on the situation ». Andersson note aussi : «The lack of consistency in the use of the term decoupling is probably partially due to a lack of a common definition of the concept, which calls for caution in the use of this term» (Andersson, 2004).

Considérant l'ambiguiité existant au sein même de la discipline économique et dans l'application qui est faite du terme découplage dans les négociations multilatérales sur le commerce, il nous a semblé pertinent de tenter de préciser, à l'aide d'experts internationaux, les éléments de définition recueillis et les principes sur lesquels bon nombre d'entre eux peuvent s'entendre concernant le concept de découplage. L'exercice, sans pouvoir servir de définition officielle du découplage, permettrait à tout le moins de proposer des frontières plus précises à ce terme si fréquemment utilisé. La démarche choisie pour valider ces propositions en est une de type Delphi. 


\section{La méthode Delphi consultation des experts internationaux}

La méthode Delphi a été développée dans les années 60 par une équipe de chercheurs dirigée par Dalkey et Helmer. C'est un processus structuré et interactif visant à recueillir les avis ou les connaissances d'un groupe d'experts (Adler et Ziglio, 1996). Plus précisément, elle consiste à soumettre des questionnaires successifs à un groupe d'experts en visant à structurer un processus de communication de groupe, sans l'interférence du comportement social interactif qui peut se produire dans une discussion face à face (Wissema, 1982). Après chaque ronde de questions, un résumé anonyme de l'opinion ou de la prédiction des experts ainsi que de leurs justifications est préparé par le facilitateur et retourné à ces derniers. Les experts sont alors encouragés à réviser ou justifier leurs réponses initiales à la lumière de l'information provenant des autres experts. Ce processus favorise une certaine convergence des réponses et le processus se termine lorsqu'il est jugé qu'un certain niveau de consensus est atteint entre les experts ou qu'un niveau de saturation dans les réponses est atteint (Mayer et Ouellette, 1991). Notre application se situe au niveau du Delphi argumentaire (Argument Delphi) développé par Osmo Kuusi ; cette approche favorise la discussion et vise à faire ressortir les arguments pertinents plutôt que la seule recherche de consensus. Cette technique est particulièrement appropriée pour les besoins de cette analyse étant donné la complexité du problème sous étude et la distance géographique qui sépare les experts retenus, puisque ceux-ci proviennent à la fois d'Europe, du Canada et des États-Unis. Les experts choisis pour cette consultation devaient avoir une scolarité de troisième cycle en économie et avoir publié ou être chercheur dans le domaine du commerce international. La consultation s'est déroulée du début novembre 2007 à la mijanvier 2008.
Concrètement, le premier questionnaire était conçu pour valider, à l'aide des experts, les éléments recueillis dans la littérature quant aux frontières possibles du concept de découplage. Lors du deuxième questionnaire nous avons présenté les résultats du premier questionnaire, soit un résumé et une analyse des éléments sur lesquels il y avait consensus ou pas. Nous avons relancé certaines questions pour préciser des éléments ou pour permettre à l'ensemble des participants de s'exprimer sur des conclusions tirées du matériel qui nous avait été transmis. Après ces deux tours, un consensus a été pratiquement atteint.

Les règles de consultation de la méthode Delphi garantissent l'anonymat des experts consultés. Cependant, il apparaît tout de même important de noter que les cinq experts ayant accepté de participer à l'étude provenaient du Québec, de France, d'Espagne, des États-Unis et du Royaume-Uni. Ces experts ont donc eu l'occasion d'échanger entre eux sur une base anonyme tout en disposant d'une importante somme d'informations sur le concept, via la technique Delphi.

\section{Résultats de la consultation}

Nous pouvons résumer les précisions apportées par les chercheurs en sept principaux éléments. Nous les reprenons un à un en discutant directement de leur incidence par rapport au concept de découplage.

\section{RÉSULTAT 1}

C'est au regard de la production que le découplage des aides aux revenus doit s'appliquer. Les effets des politiques sur la production sont au centre du débat sur le découplage.

Lorsque nous avons présenté aux experts les différents éléments de définition recueillis dans la revue de littérature, nous leur avons demandé de les classer par ordre de pertinence. Nous avons obtenu un 
consensus au sein des participants sur le fait que, du moins conceptuellement, le découplage des aides aux revenus ne s'applique qu'à la production. Les éléments de définition suivants ont également fait consensus parmi les experts quant à leur pertinence :

- Pas de variation d'allocation des ressources.

- Libre détermination des prix de marché.

- Pas de lien direct avec les prix courants.

- Pas de poids sur les décisions de production.

- Pas de distorsion de la production.

Cette précision est importante, puisqu'elle circonscrit fortement les frontières du concept par rapport aux différentes définitions recueillies dans la littérature.

\section{RÉSULTAT 2}

La demande et la consommation ne sont pas des déterminants importants dans le concept de découplage.

Cette affirmation complète le résultat précédent et est, elle aussi, intéressante, puisque plusieurs auteurs faisaient a contrario référence au découplage comme une politique n'ayant pas d'effets sur la consommation, donc sur la demande des consommateurs.

\section{RÉSULTAT 3}

Le concept de découplage n'est pas relié à la notion de variation des échanges commerciaux. La non-variation des échanges est un indicateur indirect qui découle de la non-variation de la production, mais cet élément ne fait pas partie de la définition économique du découplage.

Les éléments de définition portant sur le commerce et les échanges n'ont pas obtenu de consensus ferme au sein des experts consultés, cela démontre bien l'ambivalence de certains concernant la mesure de la non-distorsion. Tel que le note un des experts participants, "la notion de découplage n'est pas directement liée aux échanges, quoique le découplage soit un indicateur de 'non-distorsion de concurrence' dans l'esprit de certains ».

Ainsi, la non-variation des échanges et du commerce peut faire office de mesure du découplage d'une politique, mais ce ne sont pas des éléments de définition reliés directement au concept économique.

\section{RÉSULTAT 4}

L'approche par critères (ex-ante) permet de rendre le concept opérationnel. Compte tenu de l'Organe de règlement des différends utilisé dans le cadre de l'OMC, une approche ex-ante dans la définition de ce qui est découplé ou non semble être la seule option gérable.

Les experts se sont entendus sur le fait qu'il était plus réaliste d'utiliser une approche ex-ante pour définir le découplage, même si celle-ci ne permet pas de mesure empirique, contrairement à l'approche expost, et ne garantit pas nécessairement la non-distorsion sur la production. Plusieurs répondants ont noté que l'approche par critères ne pouvait rendre compte de la complexité des effets des paiements de soutien des revenus sur les choix de production. Aussi cette définition ne serait qu'approximative, étant donné la complexité du processus de prise de décision dans un monde d'incertitude où les anticipations jouent un rôle important. L'utilisation du concept dans les négociations multilatérales sur le commerce et éventuellement par l'Organe de règlement des différends se doit d'être réalisée à l'aide de critères. Normalement, ceux-ci devraient être établis en fonction des effets attendus d'une politique découplée, au sens économique ; même si ces effets ne pourront pas, à toute fin pratique, être mesurés empiriquement.

Ce résultat est cohérent avec le fait que dans les d'ouvrages consultés lors de la revue de littérature, certains auteurs (notam- 
ment Swinbank et Tranter, 2005) font référence directement à la définition du découplage de l'OMC pour définir le concept.

\section{RÉSULTAT 5}

- Le découplage est un concept relatif. Dans la réalité, le découplage total n'existe pas. Sur un horizon de long terme, toutes les politiques sont couplées. Pour être complètement découplée, une politique devrait engendrer la même réponse en termes de production à la suite d'un choc observé sur le marché. Or, il y aura toujours des effets, notamment les effets de diminution du risque, des effets de substitution, et des effets d'investissement entraînés par le maintien ou la mise en place d'une politique de revenus. Il est quasi impossible qu'une telle politique n'entraîne aucun effet.

- Une politique est découplée de la production si elle n'entraîne pas d'effets directs sur celle-ci. Comme sur un horizon de long terme toutes les politiques ont des effets et tout est sujet à changement, on considère les effets à court terme et moyen terme pour déterminer si une politique a des impacts sur la production.

Lors de la deuxième ronde Delphi, nous avons notamment demandé aux experts de s'exprimer sur la possibilité de mettre en place une politique totalement découplée. Il y a eu consensus sur le fait que le découplage est un concept relatif, puisqu'il est difficile d'imaginer, dans la réalité, la mise en place d'une politique qui n'aurait absolument aucun impact sur le coût marginal des producteurs. L'existence de paiements totalement découplés de la production (c'est-à-dire aptes à redistribuer le revenu entre groupes d'agents sans interférer avec la prise de décision concernant la production) est donc théorique. Comme il est impossible d'intervenir sans modifier le coût marginal, le découplage total n'existe pas. À ce titre les résultats du Delphi font écho au contenu d'une partie de la littéra- ture sur le découplage.

Enfin, les experts ont précisé qu'une politique pourrait être considérée comme étant découplée et ce, même si elle avait des effets (d'assurance, de risque, etc.) sur la production, mais en autant que ceux-ci ne soient pas directs (en les considérant sur un horizon de court terme). Ce résultat peut questionner le concept de plusieurs façons et nous amène à en préciser davantage les frontières.

Le découplage serait-il donc ainsi relatif à une intensité du « couplage »des aides et non pas à la suppression d'un couplage ? Si on considère que les effets directs sont les seuls à analyser pour définir ce qui est couplé ou non, mais que l'on permet à une politique découplée d'avoir des effets d'assurance et de risque, le découplage n'est-il que la suppression du lien entre le nombre d'unités produites et la subvention des revenus? Dans ce cas, les objectifs du découplage, au sens économique du terme, sont particulièrement réduits par rapport à la diversité des éléments soulevés dans la littérature et aux diverses compréhensions du terme au sein même de la profession d'économiste.

\section{RÉSULTAT 6}

- L'approche ex-post serait plus précise pour définir économiquement le découplage. Examiner les effets d'une politique sur la production permettrait de mesurer un degré de découplage, puisque certaines politiques sont plus découplées que d'autres. Toutefois, l'utilisation de degrés de découplage serait très difficile dans un cadre légal comme l'OMC. - Si on vise à mieux étudier le découplage et tendre vers une intervention étatique causant le moins de distorsion possible sur la production, l'approche ex-post demeure intéressante pour faire avancer les connaissances avec des données empiriques. 
Les experts consultés se sont entendus sur le fait que l'approche ex-post, c'est-àdire de définir le découplage au moyen des effets engendrés par les politiques agricoles, permettrait d'obtenir une définition économique plus précise du concept que l'approche ex-ante, et de pouvoir le mesurer. Par contre, les mêmes experts ont conclu, tel que rapporté au résultat 4 cidessus, que seule l'approche ex-ante peutêtre applicable dans la réalité des ententes multilatérales sur le commerce. L'approche ex-post serait tout de même utile pour permettre de déterminer le degré de découplage d'une politique agricole, puisqu'elle permet de fournir des données empiriques, ce que l'approche ex-ante ne permet pas.

Si le découplage total n'existe pas dans la réalité, mais qu'on considère que les politiques plus découplées de la production demeurent plus pertinentes (en termes d'efficacité de transfert et de moindre impact de distorsion des décisions de production), il serait intéressant de mesurer empiriquement le plus ou moins grand découplage des aides. Pour rendre le concept plus opérationnel, le découplage peut être considéré en termes de degré plutôt qu'en valeur absolue. Il semble donc qu'une politique qui aurait des effets minimes mais non nuls sur la production pourrait ainsi être considérée comme "découplée » au sens économique du terme.

Ceci fait d'ailleurs écho à certains écrits qui classent les outils d'intervention en termes de soutien des revenus aux agriculteurs selon leur efficacité de transfert et leur « préférabilité ». Les études sur le découplage tendent ainsi à démontrer que le découplage, même partiel, induit moins de distorsion qu'une politique couplée (Anton et Sckokai, 2006).

\section{Conclusion}

L'utilisation des mesures couplées à la production a été, et est toujours, très importante dans le choix des instruments d'intervention publique en agriculture ${ }^{4}$. La mise en place, au fil du temps, de politiques de soutien des revenus lié a, elle aussi, suscité de vives critiques, notamment en ce qui concerne leurs effets néfastes sur l'offre.

La notion de découplage des aides aux revenus est donc apparue dans les débats publics et économiques, au cœur d'une recherche vers une meilleure efficience des instruments de la politique agricole. Si les préoccupations et les propositions de dissocier le soutien de l'État des produits sont présentes dans la littérature économique depuis fort longtemps, c'est surtout depuis le début du Cycle de l'Uruguay que le terme découplage est fréquemment utilisé. De ce point de vue, le concept de découplage devrait permettre de mettre en place des politiques qui répondent, du moins partiellement, aux préoccupations qui ont amené le concept au cœur des débats.

L'analyse de la littérature économique permet de constater le peu de consensus qui existe quant au concept de découplage. Ce constat nous a amené à vouloir clarifier le concept à l'aide de la méthode Delphi. Nonobstant le nombre relativement restreint de participants, cet exercice a permis d'identifier les frontières entourant la définition du découplage et d'en préciser la définition. Les éléments sur lesquels les experts se sont entendus ont permis de valider nos deux propositions initiales et peuvent être résumés en sept éléments principaux :

1. C'est au regard de la production que le découplage des aides aux revenus doit s'appliquer. On considère une politique découplée si elle n'entraîne pas d'effets directs sur la production.

4. En 2005, l'OCDE estime qu'en moyenne près de $54 \%$ de l'estimation du soutien au producteur dans ses pays membres est composé de mesures de soutien des prix du marché (OCDE, 2006). Il faut ajouter à cette estimation les mesures de soutien du revenu lié, ce qui fait qu'une large part du soutien reste couplée. 
2. La demande et la consommation ne sont pas des déterminants importants dans le concept de découplage.

3. La non-variation des échanges est un indicateur indirect du découplage qui découle de la non-variation de la production, mais cet élément ne fait pas partie de la définition économique du découplage.

4. L'approche par critères (ex-ante) est plus réaliste pour l'obtention d'une définition économique. Elle permet de rendre le concept opérationnel.

5. L'approche ex-post serait plus précise pour définir économiquement le découplage. Examiner les effets d'une politique sur la production permettrait de mesurer un degré de découplage.

6. Le découplage est un concept relatif. Le découplage total n'existe pas. On peut toutefois envisager de mesurer un degré de découplage.
Finalement, le découplage des aides aux revenus de la production n'est peut-être pas, a priori, un concept économique à part entière mais bien un amalgame de critères, une stratégie pouvant mener à une intervention de l'État à la fois plus précise pour les bénéficiaires (en termes de réduction de la variabilité des revenus et pour contrer leur faiblesse relative), et préférable en terme de bien-être pour la société. Enfin, le remaniement des aides aux revenus intervient également dans une optique plus large, celle de les rendre plus acceptables et plus justifiables pour les différents partenaires commerciaux, dans un système commercial maintenant fortement globalisé. Le découplage des aides aux revenus de la production peut à ce titre être considéré comme un pas dans la recherche de nouveaux paradigmes et de nouveaux modèles d'intervention de l'État pour le développement de l'agriculture.

\section{RÉFÉRENCES BIBLIOGRAPHIQUES}

Adler M., Ziglio E. (1996). Gazing into the Oracle. Bristol, Jessica Kingsley Publishers, PA.

Andersson F. (2004). Decoupling: the concept and past experiences. Suède, Swedish Institute for food ans, Agricultural Economics, 50 p.

Antón J., Sckokai P. (2006). La difficulté de découpler les soutiens à l'agriculture. EuroChoices, vol. 5, n 3, p. 13-19.

Beard N., Swinbank A. (2001). Decoupled payments to facilitate CAP reform. Food Policy, ${ }^{\circ} 26$, p. 121-145.

Bergmann D. (1980). Possible alternatives to the CAP and their economic consequences. Cité dans « Swinbank et Tranter» (2004). London, Paper at the conference of the Agricultural Economics Society on the CAP in the 1980's at the Royal Society.

Boschwitz R. (1987). Decouple in the long run. Choices, Third Quarter.
Brandow G.-E. (1955). A modified compensatory price program for agriculture. Cité dans Gilson (1988), Journal of Farm Economics, november, p. 717-718.

Burfisher M.-E., Hopkins J. (2003). Decoupled payments: Household income transfers in contemporary U.S agriculture. Agricultural Economic Report no.822, Economic Research Service, United State Department of Agriculture (USDA), 29 p.

Burfisher M.-E., Hopkins J. (2004). Decoupled payments in a changing policy setting. United State Department of Agriculture, USDA, Agricultural Economic Report no.838, Economic Research Service, $61 \mathrm{p}$.

Butault J.-P., Le Mouël C. (2004). Pourquoi et comment intervenir en agriculture? In Butault J.-P. (éditeur) «Les Soutiens à 
l'agriculture, théorie, histoire, mesure », Paris, INRA Éditions, p. 11-67.

Cahill S.-A. (1997). Calculating the rate of decoupling for crops under CAP/oilseeds reform. Journal of Agricultural Economics, vol. 48, n 3 , p. 349-378.

Carr A.-B., Rossmiller G.-E. (1988). Un point de vue américain sur le découplage. In Finkle P., Cameron D. (éd.) «Le découplage au Canada: perspectives d'avenir », Canada, Institut Canadien des ressources juridiques et Agriculture, p. 153-165.

Collins K., Vertrees J. (1988). Decoupling and U.S. farm policy reform. Canadian Journal of Agricultural Economics, vol. 36, n 4, p. 733-745.

Finkle P., Cameron D. (1988). Le découplage au Canada : perspectives d'avenir. Canada, Institut Canadien des ressources juridiques et Agriculture, $182 \mathrm{p}$.

Gilson J.-C. (1988). Premières expériences dans le domaine du couplage et du découplage des programmes de soutien des prix agricoles et des produits agricoles primaires. In Finkle P., Cameron D. (éd.) "Le découplage au Canada: perspectives d'avenir ». Canada, Institut Canadien des ressources juridiques et Agriculture, p. 7-14.

Gohin A., Gorin O., Guyomard H., Le Mouël. C. (1999). Interprétation économique, avantages et limites du principe de découplage des instruments de soutien des revenues agricoles. Notes et études économiques, vol. 10, INRA, p. 9-37.

Gouin D.-M. (2004). La gestion de l'offre dans le secteur laitier: un mode de régulation toujours pertinent. Groupe de recherche en économie et politique agricoles (GREPA), Université Laval, Département d'économie agroalimentaire et des sciences de la consommation, $134 \mathrm{p}$.

Gouin D.-M. (2005). La performance économique comparée des systèmes de régulation du secteur laitier, une analyse internationale. In « Notes et études économiques ». Paris, Sous-direction de l'évaluation, de la prospective et des études, ministère de l'Agriculture, de l'Alimentation, de la Pêche et de la Ruralité, $n^{\circ}$ 24, décembre, p. 99-133.

Guyomard H., Bureau J.-C., Gohin A., Le Mouël C. (2000). Impact of the 1996 US FAIR ACT on the Common Agricultural Policy in the World Trade Organisation context: the decoupling issue. Food Policy, vol. 25, $\mathrm{n}^{\circ}$ 1, p. 17-34.

Hathaway D.-E. (1968). Government and agriculture: economic policy in a democratic society. New-York, The Macmillan Compagny, 412 p.

Hennessy D.-A.(1998). The production effects of agricultural income support policies under uncertainty. American Journal of Agricultural economics 80, p. 46-57.

Koester U., Tangermann S. (1977). Supplementing farm price policy by direct income payments: cost benefit analysis of alternative farm policies with a special application of German agriculture. Cité dans « Swinbank et Tranter» (2004). European Review of Agricultural Economics, n $^{\circ}$ 4, p. 7-31.

Little J., Knapp T., Sottomayor M., Swinbank A. (2004). Compensation Proposals for EU Farm Policy Reform. Cité dans « Swinbank et Tranter » (2004), Chapitre 3, p. 39-53.

Mayer R., Ouellette F. (1991). Méthodologie de recherche pour les intervenants sociaux. Boucherville, Éditions Gaëtan Morin.

Morin-Rivet L. (2008). Le découplage des aides aux revenus: un concept économique imprécis. Mémoire de maîtrise, Université Laval, Québec.

Moschini G., Sckokai. P. (1994). Efficiency of decoupled farm program under distortionary taxation. American Journal of Agricultural Economics, vol. 76, $\mathrm{n}^{\circ} 3$, p. 362-370.

Nicholls W.-H, Johnson D.-G. (1946). The farm price policy awards. Journal of Farm Economics, février, p. 28. 
OCDE (2001). Découplage : une vue d'ensemble du concept. Paris, Document de l'OCDE, n 10, 45 p. http://www. oecd.org/dataoecd/23/49/25485371.pdf

OCDE (2007). Le découplage du soutien à l'agriculture. Paris, Synthèses, L'Observateur OCDE, 8 p.

OMC (2006). Annexe 2 de l'AAUR, Soutien interne: base de l'exemption des engagements de réduction. Site Internet de l'OMC, consulté le 15 août, http://www.wto.org/french/docs f/legal f/ 14 ag.pdf

Quaden G. (1973). Parité pour l'agriculture et disparités entre agriculteurs. Essai critique sur la politique des revenus agricoles. Collection scientifique de la Faculté de Droit de l'Université de Liège, $236 \mathrm{p}$.

Ray D.-E. (2000). Payments are decoupled from production but is production decoupled from payments? Policy Pennings, Agricultural Policy Analysis Center, sept., University of Tennessee, 1 p. http://agpolicy.org/weekcol/011.html

Ray D.-E. (2002). Yes, decoupled, but decoupled from what? Policy Pennings, nov., Agricultural Policy Analysis Center, University of Tenessee, $1 \mathrm{p}$. http://agpolicy.org/weekcol/117.htm

Revell B., Oglethorpe D. (2003). Decoupling and UK agriculture: a whole farm approach. Royaume-Uni, DEFRA, Harper Adams University et SAC Edinburgh, $17 \mathrm{p}$.

Roberts I., Podbury T., Freeman F., Tielu A., Vanzetti D., Andrews A., Melanie J.-A.,
Hinchy M. (1999). Reforming world Agricultural trade policies. Cité dans Swinbank and Tranter (2004), ABARE Research Report 99.12, Australian Bureau of Agricultural and Ressource Economics, Canberra.

Schmitz A., Furtan H., Baylis K. (2003). Agricultural Policy, Agribusiness and rent-Seeking Behaviour. Toronto, University of Toronto press, $511 \mathrm{p}$.

Shepherd G.-S. (1964). Farm Policy: New Directions. Ames, Iowa, Iowa State University Press, p. 169.

Swerling B.-C. (1959). Income protection for farmers: a possible approach. Cité par Gilson (1988) et Hathaway (1968), Journal of Political Economy, avril, p. 173-186.

Swinbank A., Tranter R. (2004). A bond sheme for the Common Agricultural Policy reform. Royaume-Uni, Wallingford, Center for Agricultural Strategy, University of Reading, CABI, 187 p.

Swinbank A., Tranter R. (2005). Decoupling EU farm support: does the new single payment scheme fit within the green box? The Estey Center Journal of International and Trade Policy, vol. 6, n ${ }^{\circ}$, p. 47-61.

Warley T.-K. (1988). Avant-propos. Finkle P., Cameron D. (éd.). In « Le découplage au Canada: perspectives d'avenir », Canada, Institut Canadien des ressources juridiques et Agriculture, p. $1-5$.

Wissema J.-G. (1982). Trend in technology forecasting. Research and Development Management, vol. $12 \mathrm{n}^{\circ}$ 1, p. 27-36. 